\title{
CBL+TBL 混合教学模式在大学计算机教学中的应用初探
}

\author{
张忠梅 \\ 江西科技师范大学 \\ DOI:10.12238/mef.v3i9.2885
}

\begin{abstract}
[摘 要] 大学计算机教学随着信息技术的发展, 伴随着历次教育体制改革而不断变革, 取得了一些成 效, 也在历次变革中积累了一些经验。但在当前应用型人才培养背景下, 现有的教学体系还存在着许 多与新形势、新任务、新需求不相适应的地方，如教学观念、体制、体系、方法、手段等。为适应新 形势的需要, 本文从分析当前教学的现状出发, 提出案例教学和团队教学的混合教改新模式, 并对该 模式的理论依据、实施细则和效能优势进行系统的阐述。
\end{abstract}

[关键词] 案例教学模式 ( CBL); 团队教学模式 ( TBL); 混合教学模式

中图分类号: G64 文献标识码: A

\section{A Probe into the Application of CBL+TBL Hybrid Teaching Model in College Computer Teaching Zhongmei Zhang \\ Jiangxi Science and Technology Normal University}

[Abstract] With the development of information technology and the reform of educational system, College Computer Teaching has made some achievements and accumulated some experiences. However, under the background of current applied talents training, the existing teaching system still has many places that are not compatible with the new situation, new tasks, and new needs, such as teaching concept, system, methods and means. In order to meet the needs of the new situation, starting from the analysis of the current situation of teaching, this paper puts forward a new hybrid teaching model of case teaching and team teaching, and systematically expounds the theoretical basis, implementation rules and efficiency advantages of the model.

[Key words] Case-Based Learning(CBL); Team-Based Learning(TBL); hybrid teaching model

随着科学技术的迅猛发展, 以计算 机为核心的信息技术在人们的学习、工 作和生活中发挥着越来越重要的作用。 计算机成为生活中一种必不可少的工 具, 在提高学生综合素质, 培养学生创 新能力等方面扮演着越来越重要的角 色。计算机主要考查学生的实际操作能 力, 尤其是计算机基础课程的很多知识, 如果仅仅靠教师课堂讲解和演示操作, 学生遇到真正的问题依然一片茫然, 只 有经过亲自实践, 并在实践操作中分析 解决遇到的问题, 才能提高学生的综合 实践能力。大学计算机教学随着信息技 术的发展, 伴随着历次教育体制改革而 不断变革, 取得了一些成效, 也在历次 变革中积累了一些经验。但在当前应用 型人才培养背景下, 现有的教学体系还
存在着许多与新形势、新任务、新需求 不相适应的地方, 如教学观念、体制、 体系、方法、手段等。为适应新形势的 需要, 进一步推进计算机教学改革, 建 立一套系统化、条理化、集成化的全新 理论, 指导大学计算机教学实践, 推进 计算机实验教学水平跃上新台阶已成当 务之急。

\section{1 当前大学计算机教学的现状}

我国许多大学的计算机教学一直以 来沿用的教学模式是, 先由教师讲解原 理、操作步骤软件的使用情况等, 学生 再按老师和教材所讲重复实践。这种教 学模式弊端多、效果差。学生在学习过 程中, 只是填鸭式地被动接受老师传授 的知识, 学习兴趣不浓厚, 厌学情绪在 学生中滋长, 学生不愿也不想去动手操
作，更别谈创新能力的培养了。鉴于当 前传统计算机教学模式的弊端, 改革势 在必行。目前, 国内外很多学者意识到 了问题, 并进行了相关研究, 也提出了 一些新的教学模式, 如开放型实践教学 模式、分层型实践教学模式等, 这些教 学模式对于培养学生的能力, 提高学生 的素质来说是非常有效的, 但由于教学 资源有限，师资短缺，且技术难度大， 因而操作的普适性不强, 只能作为一个 长远的发展方向努力争取。

\section{CBL+TBL 混合教学模式的涵义}

案例教学模式 (Cased-Based Learning) 是有问题式教学模式发展而来的, 以案 例为基础, 选取典型的案例, 设计与之 相关的问题, 引导并启发学生围绕问题 展开讨论的一种讨论式教学法。这种教 
学模式在医学教学中使用较为广泛。

团队教学模式 (Team-Based Learning) 核心理念是小组成员间的团队合作，他 们是以小组为基础, 将全班的学生分成 一个个小组, 小组人数不宜过多, 过多 则不能很好地发挥每位成员的作用，过 少则每个成员的任务较多, 又无法对任 务进行深入思考, 根据实际情况 $4-5$ 人为 一小组较为合适, 并将每个小组看作一 个团队, 团队成员自主学习, 团队内合 作完成老师布置的一个个学习任务。该 教学模式在美国等发达国家得到了广泛 的应用, 并取得了较好的教学效果, 我 国部分医学院校也运用团队教学模式来 推动教学改革。团队教学模式的学习方 式是自主学习和组内与组间讨论相结 合。团队成员根据老师布置的实验内容 分工合作, 准备相关资料, 实验课上通 过组内探究、组间讨论和教师引导, 合 作完成实验内容，撰写实验报告。

我们将案例教学模式和团队教学模 式引入到大学计算机实验教学中来, 以 案例教学为基础, 将成员分成一个个小 团队，通过团队合作来完成老师布置的 学习任务。选取典型的案例从用户的需 求、任务概述到成本的核算等方面进行 分析, 探讨该案例的优缺点, 以及改进 的措施。

\section{$3 \mathrm{CBL}+\mathrm{TBL}$ 混合教学模式在计} 算机实验教学中的设计与实施

CBL+TBL混合教学模式的特征是以 案例为主线, 教师为主导, 学生团队为 主体, 是教师、学生、教学任务三者的 有机结合, 缺一不可。教师根据教学目 标和学生实际情况选取合适的案例, 学 生根据教师给出的案例, 团队成员间分 工合作，通过查找资料、讨论等方式对 案例进行分析、学习, 对于学习中遇到 的问题采取组内和组间讨论、教师讲解 等方式一个个的解决, 一个任务完成后 接着进行下一个任务, 直到完成整个学 习任务。在学术进行学习、实践的过程 中, 教师对学生的进度进行监控, 随时 了解学生学习过程中出现的问题, 对于 个性的问题采用单个讲解的方式予以解 决, 对于共性的问题采用分组讨论的方

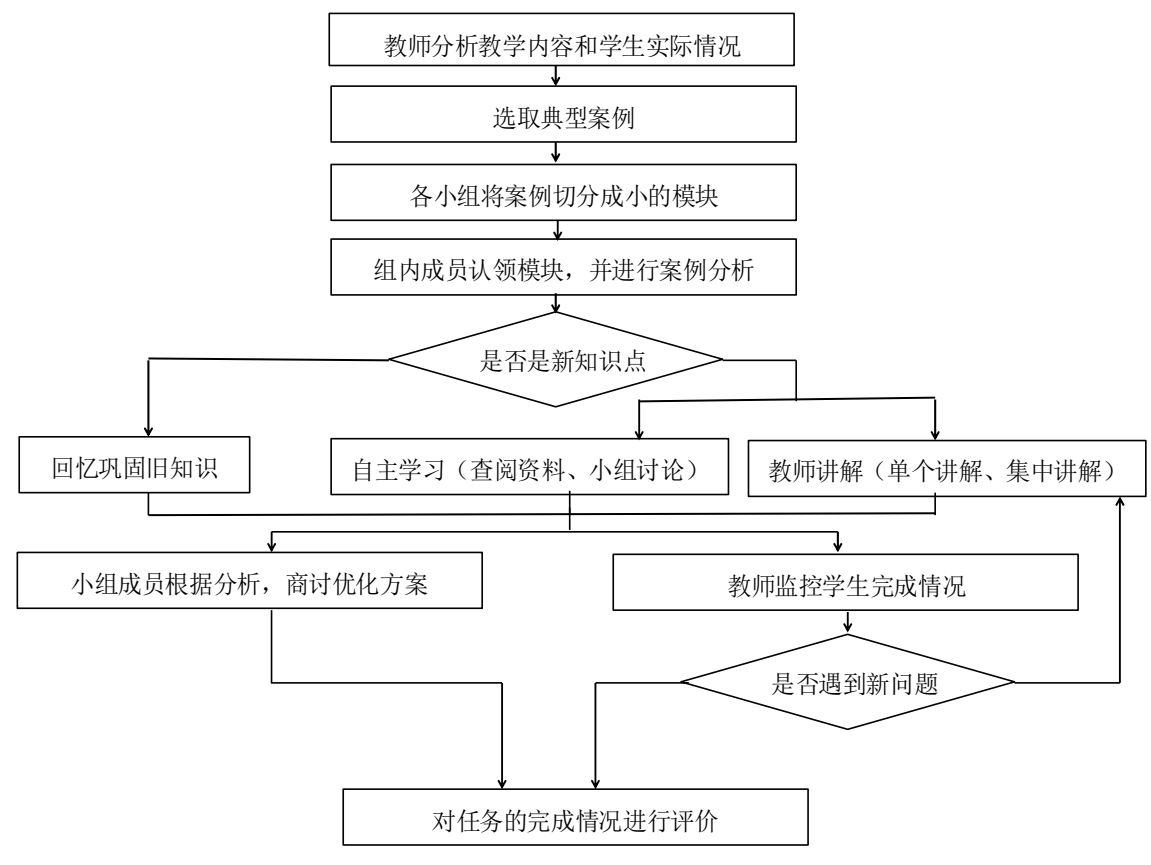

图 1 混合教学模式实施流程图

式进行引导, 使学生掌握教学目标所要 求的知识点。具体步骤如下图1所示。

案例中的一个个模块 “任务” 不等于 “问题”, 不能把一个个模块任务简单化、 平面化。案例教学法的本质是自主学习、 探究学习, 它是一个 “大容量、活模块、 多技能、有梯度” 的层级任务体系, 是一 个研究性、探究性的学习过程。

\section{$4 \mathrm{CBL}+\mathrm{TBL}$ 混合教学模式在计 算机教学中的优势}

乔纳生 (D. H. Jonasen, 1991) 曾指 出我国传统大学教学的弊端在于没有很 好地划定初级学习和高级学习的界限, 把初级阶段注重基础知识学习的教学策 略不加修改地直接搬到高级阶段注重创 新能力培养的教学过程中去, 把大学生 当作中小学生来培养, 使得教育方式过 于简单, 妨碍了学生创新能力的培养。 案例教学模式和团队教学模式的结合, 正好能纠传统教学之弊, 符合大学阶段 学习要求。其优势具体体现为:

4. 1 注重趣味性, 激发学生的学习欲 望。教师精心挑选的案例可以引起学生 的注意, 激发其主动投入到案例分析、 优化的过程中去, 在这个过程中, 学生 对案例的探索, 对案例驾驭的渴望, 就 是一种 “兴奋剂”, 使他们在学习中始终
保持浓厚的兴趣, 积极学习。

4. 2 强调真实性, 培养学生的自主学 习能力。案例教学模式使学生处在尽可能 真实的学习情境中, 学生就是案例中的真 实主体, 使更加学习直观化、形象化。当 分析完案例中的一个模块后, 成就感驱使 他们对该模块提出新的优化措施。

4. 3鼓励主体性, 培养学生的创新 能力。案例教学法是一种以学生为主体 的教学方法, 学生置身于当前案例中, 作为案例中真实存在的个体, 需要解决 案例中出现的一个个问题, 彻底摒弃了 传统教学中的 “传递一一接受” 模式, 老师不需要填鸭式地告诉学生第一步 怎么做, 第二步怎么做, 而是让学生自 己思考每一步应该怎么做, 并对自己所 做的每一步进行分析评估, 根据自己的 分析评价, 自由选择解决问题的方法和 途径。

4. 4 突出协调性, 培养学生的合作能 力。教师进行教学设计时, 将案例切分 成以一个模块, 各个模块既是独立存在 的, 又需要协作完成, 所以学生在完成 的过程中，不仅要与教师交流，还要与 团队成员之间探讨, 调整完善自己的观 点, 促进同学间良好的人际关系, 进一 步培养了学生的协作能力。 


\title{
教育创新之浅谈学习兴趣培养的重要性
}

\author{
程丽华 \\ 天津市南开区东方小学 \\ DOI:10.12238/mef.v3i9.2911
}

\begin{abstract}
[摘 要] 本文以老子的 “授之以鱼, 不如授之以渔” 为入题点, 引出 “授之以渔, 不如授以 “欲”” 的 观点。阐述了在现阶段的教育中应以培养学生对学习的兴趣为重点, 文中以新型教育的理论和身边事 例等来说明这一观点, 并且列举出了各种激发学生学习兴趣的方法及其注意事项。
\end{abstract}

[关键词] 求知欲; 好奇心 ; 好胜心 ; 引导

中图分类号: G4 文献标识码：A

\section{A Brief Discussion on the Importance of Cultivating Learning Interest about Education Innovation \\ Lihua Cheng \\ Dongfang Primary School, Nankai District, Tianjin}

\begin{abstract}
[Abstract] This article takes Lao Tzu's "It is better to teach him how to fish than to give him fish" as the starting point, and draws the point of view that "It's better to teach him the desire of fishing than to teach him how to fish." It states that the current education should focus on cultivating students' interest in learning. The article uses new educational theories and examples around to illustrate this point of view, and lists various methods to stimulate students' interest in learning and their attention matter.
\end{abstract}

[Key words] desire; curiosity; ambition; guidance

“授之以鱼, 不如授之以渔” 是我 国伟大思想家老子的教学观念, 他主张 不能一味地传授他人知识, 更重要的是 要传授他人获取知识的方法, 这一经典 而伟大的教育理念被我们一直沿用至 今。然而, 在现如今这个信息高速发达 的社会, 对学生, 尤其是小学的学生而 言, 他们对社会的认知程度以及对新鲜 事物的接受程度比之若干年前提升了许

\section{基金资助:}

江西科技师范大学人文社科科研计 划项目: 平安校园建设背景下大学生网络 素养培养研究 (编号: 2019XJYB0012); 江西科技师范大学教改课题: 应用型人才 培养背景下CBL+TBL混合教学模式在计 算机基础教学中的应用研究 (编号: JGYB-19-100-9)。

[参考文献]

[1]宋秋英,王晓朴.教育信息化背景
多倍, 同时, 在孩子眼中, 任何一种事 物似乎都比学习更让他们感兴趣。那么, 如何能让学生更好的, 更踏实的获取知 识呢? 无疑就是让学生首先对学习产生 兴趣。所以, 我更认为授之以渔, 不如 授之以 “欲”!

\section{1 挖掘兴趣}

作为一名小学教师, 尤其是班主任, 除了平时上课之外, 课间和午休甚至放
学以后都要和学生们在一起, 其目的我 认为不仅仅是辅导知识和照顾他们生活 上面的琐事, 更重要的是要发掘一些学 生平时在课堂上不容易表现出来的想 法, 因为只有在这些时候, 学生才是真 正的无拘无束, 我可以站在孩子的角度 与他们聊天, 融入到他们的话题当中去, 有时候, 我会发现平时连几个简单汉字 都经常记错的学生, 对自己喜爱的动画
下案例教学模式设计初探[J]. 中国教育 学刊,2020(S1):166-167.

[2]张华平, 苏欣, 陈虹羽, 等. 项目任 务驱动的案例教学改革研究与实践--以《房屋建筑学》课程为例[J]. 高教学 刊,2019(08):139-141.

[3]王洪才, 李慧.运用生成性案例实 现创新性教学[J].中国高等教育,2013 (19):44-46.

[4]郝文艺.PBL+TBL 教学模式在金
课建设的应用探索 [J]. 教育教学论 坛,2020(19):270-271.

[5]徐磊.能力导向教学法的融合研 究与实践--- 基于 "PBL+TBL" 完整行动 模式[J]. 教育观察,2019,8(16):105-107.

\section{作者简介:}

张忠梅 (1979--), 女, 汉族, 湖 北京山人, 讲师, 硕士; 研究方向: 计 算机应用技术、教育管理等。 